\title{
Maternal postpartum feeding anxiety was associated with infant feeding practices: results from the mother-infant cohort study of China
}

Jing Sun ${ }^{1}$, Yimin Zhu ${ }^{2}$, Yongjin Li ${ }^{3}$, Niuniu Li ${ }^{1}$, Tan Liu' ${ }^{1}$ Xiao Su${ }^{1}$, Zhiyong Dai ${ }^{4}$, Yanchun Zhang ${ }^{4}$, Lina Pan ${ }^{4}$, Wei Jiang ${ }^{4}$ and Wenli Zhu ${ }^{1 *}$

\begin{abstract}
Background: Maternal feeding anxiety (FA) was prevalent during puerperium and might affect infant feeding practices. This study was aimed to investigate the FA status in Chinese postpartum women and its relationship with infant feeding practices (FPs).

Methods: Participants were from the Mother-Infant Cohort Study of China, in which the dietary and feeding practices, physical and psychiatric health for both mothers and infants were followed up from childbirth to next 2 years. In this study the maternal feeding anxiety (FA) status at 0-3 months postpartum was assessed by Li's Selfrating Feeding Anxiety Scale (SFAS). Infant feeding practices (FPs) at 0-3 months, including breastfeeding-related behaviors, responsive feeding and infant food refusal were investigated by self-designed questionnaire.
\end{abstract}

Results: In total 456 mothers the average feeding anxiety scores (FAS) was $41.02 \pm 8.02$ (mean \pm SD), and maternal FA prevalence were $61.4 \%$ (FAS $>38$ ) with severe FA being $8.6 \%$ (FAS $>52$ ) at 0-3 months postpartum. The FAS was related with infant FPS, and lower maternal FAS was significantly related with infant colostrum feeding $(40.86 \pm 8.02$ vs $44.74 \pm 11.33, P<0.05)$, but higher FAS was related with bottle feeding $(41.95 \pm 8.28$ vs $39.69 \pm 7.92, P<0.05)$. The mothers with severe feeding anxiety (FAS > 53) were more likely to feed infants with bottle (ORs, 95\% $\mathrm{Cl}: 2.41,1.11$ $\sim$ 5.19). There were not significant association between FAS and exclusive breastfeeding and responsive feeding practices $(P>0.05)$. The higher FAS was associated with infant food refusal behaviors, the maternal scores whose infant "never", "rarely", "sometimes" and "often" spat out food when feeding were 39.86 $\pm 8.02,41.47 \pm 8.18,41.36 \pm$ 7.44 and $42.14 \pm 12.03$ increasingly $(P>0.05)$, and the FA prevalence was significantly different among groups $(P<$ 0.05). The infants whose mother was identified as feeding anxiety were more likely to refuse opening the mouth when feeding $(P<0.05)$. Multivariate analysis indicated maternal FAS was positively related to infant bottle feeding $(\beta i=2.487, P<0.05)$ and outdoor sunshine exposure practice $(\beta i=1.787, P<0.05)$, and negatively related to household income level $(\beta i=-0.118, P<0.05)$.

(Continued on next page)

\footnotetext{
* Correspondence: zhuwenli@bjmu.edu.cn

'Department of Nutrition and Food Hygiene, Peking University School of

Public Health; National Health Commission Key Laboratory of Reproductive Health, Beijing 100191, China

Full list of author information is available at the end of the article
}

(c) The Author(s). 2020 Open Access This article is licensed under a Creative Commons Attribution 4.0 International License, which permits use, sharing, adaptation, distribution and reproduction in any medium or format, as long as you give appropriate credit to the original author(s) and the source, provide a link to the Creative Commons licence, and indicate if changes were made. The images or other third party material in this article are included in the article's Creative Commons licence, unless indicated otherwise in a credit line to the material. If material is not included in the article's Creative Commons licence and your intended use is not permitted by statutory regulation or exceeds the permitted use, you will need to obtain permission directly from the copyright holder. To view a copy of this licence, visit http://creativecommons.org/licenses/by/4.0/ The Creative Commons Public Domain Dedication waiver (http://creativecommons.org/publicdomain/zero/1.0/) applies to the data made available in this article, unless otherwise stated in a credit line to the data. 
(Continued from previous page)

Conclusions: Maternal postpartum feeding anxiety was associated with some infant feeding practices, including bottle feeding and infant food refusal behaviors.

Keywords: Maternal, Postpartum feeding anxiety, Infant feeding practices, Breastfeeding

\section{Background}

While the physical health of women and children is emphasized, the mental aspects of their health are often ignored, especially in low- and middle-income countries (LMICs). Literatures showed that perinatal anxiety and depression are more prevalent in women from LMICs, being $15.6 \%$ during the prenatal period and $19.8 \%$ during the postnatal period, as compared to high-income countries, ranging from 6.5 to $12.9 \%$ during the perinatal period [1]. Globally, in women of childbearing age, postpartum psychiatric symptoms including anxiety and depression account for the largest proportion of the burden associated with mental or neurological disorders. In addition to the economic and human costs of maternal depression, children of mothers who are depressed are at risk for poor health, developmental, and behavioral problems, thereby contributing to intergenerational disadvantage that accumulates throughout the life span.

The aetiology of perinatal mental health problems is multifactorial, with studies consistently reporting the importance of psychosocial variables such as life stress and lack of social support. In China postpartum women participate in a traditional ancient practice called "zuoyuezi" or "doing the month", including special diet, maintaining warmth, restricting physical and outdoor activity. During the puerperium, not only the limited socialization, but also the contradiction of adherence to traditional beliefs supported by family elders and modern scientific guideline, may increase the postpartum psychiatric symptoms risk in Chinese women. A longitudinal cohort study on postpartum depression and anxiety among 571 Chinese immigrant and nonimmigrant women with live births in 2011 to 2014 showed that Chinese immigrant women are a high-risk group for postpartum depressive and anxiety symptomatology, possible depressive symptomology was most prevalent and occurred in a quarter of women at 4 weeks postpartum (24.4\%; 95\% CI, 21.1-28.2\%) and decreased to $17.9 \%(95 \%$ CI, $14.6-21.8 \%)$ at 52 weeks postpartum [2].

Poor maternal mental health is not only detrimental to the mother's physical and psychological health, but also has adverse impact on her infant's feeding practices (FPs), and ultimate growth and development. Feeding anxiety (FA) refers to an unpleasant emotional state correlated with infant FPs and outcome, which is a dimension of postnatal anxiety. FA also diminishes maternal breastfeeding confidence and self-efficacy, and can lead to a reduced duration of breastfeeding or a decline in exclusive breastfeeding $[3,4]$. A systematic review provided evidence for the effect of negative postpartum mood on breastfeeding, showed that women with symptoms of postpartum anxiety are less likely to breastfeed exclusively and initiate breastfeeding, and more likely to terminate breastfeeding earlier and supplement with formula in the hospital [5]. Heterogeneous outcomes and methodological limitations somewhat limit the comparability of findings.

Overall, The first 3 months after delivery is a critical window period for the infants, during which the maternal emotional status directly affect infant's feeding behaviors, physical and mental development, and furtherly the adulthood health $[4,6]$. It is therefore highly importance to identify and improve maternal FA to avoid its negative effects on infant FPs and development. But to now FA was rarely concerned in China. The aim of this study was to investigate the FA status in Chinese puerperal women and its relationship with infant FPs, to provide the targets for further maternal mental health program.

\section{Methods \\ Participants}

This study used data collected as part of the MotherInfant Cohort Study (MICS) of China. The MICS was conducted from June 2015 to December 2018 in seven cities (Beijing, Taiyuan of Shanxi, Jinan of Shandong, Changsha of Hunan, Shiyan of Hubei, Chongqing, and Chengdu of Sichuan) from northern, eastern, central and southwestern districts around China. At baseline motherinfant pairs at $0-3$ months postpartum were voluntarily recruited from local child care clinics, and then followed up to 2 years. The mothers were healthy and the neonates were born without severe deformities. Participants with incomplete information or logical error were removed, and 490 mother-infant pairs were included in the study and 456 questionnaires (93.1\%) were available for the final analysis. The data of the study was investigated at $0-3$ months postpartum.

The study was approved by the Peking University Institutional Review Board (Beijing, China) and informed written consent was obtained from all mother participants.

\section{Data collection}

Maternal feeding anxiety (FA) assessment

Maternal FA was assessed using Li's Self-rating Feeding Anxiety Scale (SFAS) [7] at 0-3 months postpartum. 
The SFAS (2013) scale consists of four dimensions (worrying, self-confidence, annoying and stress) and 23 items. The mothers were asked some infant feedingrelated questions, like "I was annoyed by infant's crying." "I was competent to be a mother." "I worried about child appetite." "I was stressed about infant food safety." and so on. All items are rated on a 4-point Likert scale to produce a summative score ranging from 23 to 92, with higher scores indicating increased anxiety symptom. The SFAS has been used broadly in studies of Chinese postpartum women's feeding anxiety, while a recommended cutoff of 38 [8]. The reliability and validity of SFAS were tested and Cronbach's alpha coefficient was 0.829 and the internal consistency coefficient was 0.730. In this study we defined FAS $>38$ being mild feeding anxiety and FAS $>53$ being severe anxiety (Supplementary file 1).

\section{Infant feeding practices (FPs) investigation}

Infant feeding practices (FPs) were defined as a series of behaviors and related practices exhibited by infants and mothers, that affected infant's food and nutrients intake directly or indirectly, including feeding pattern, bottle feeding, responsive feeding, mother-child interactions, food refusal, etc.

The FPs investigated in the study included: 1) Breastfeeding related practices, including touching breast in mother's arms within $1 \mathrm{~h}$ after delivery; colostrum feeding (breastfeeding within 7 days after delivery); feeding other food or liquid (excluding milk) before breastfeeding within 3 days after delivery; exclusive breastfeeding (with allowance for oral rehydration salts, vitamins, minerals, medications, and infusion); feeding water, beverages, or juice; feeding infant formula; bottle feeding. 2) Responsive feeding practices, including perception of infant hungry and satiety signs, on-demand feeding, fixed-time feeding. 3) Infant's food refusal behaviors, including refusal to open the mouth, turning head aside, crying when feeding. 4) Outdoor sunshine exposure of infant. Mothers answered the self-designed questionnaire at $0-3$ months postpartum and the options included different levels like "never", "rarely", "sometimes", "often", and "yes" or "no" (Supplementary file 2).

\section{Covariates}

Demographic characteristics were measured at the 0-3 months postpartum interview, including maternal age, child-bearing number, marital status, education level, household income (monthly income less than 3000 yuan per capita was defined as "low" level, 3000-6000 yuan being "middle", more than 6000 yuan being "high"). Gestational status and delivery mode were also investigated by questionnaire (Supplementary file 3).

Local physicians and nurses distributed the selfreported questionnaire to the women at $0-3$ months postpartum, provided explanations face to face or by phone, and guided them to fill out the questionnaire.

\section{Statistical analysis}

The EpiData3.1 software was used for data input and the SPSS 20.0 software was used for statistical analysis. Prevalence and score (mean \pm SD) of maternal feeding anxiety were calculated. Differences of FAS and FA prevalence were compared among different demographic characteristics and gestational and delivery status using $\mathrm{t}$-test, one-way analysis of variance and chi-squared test. To analyze the relationship between maternal FA and infant FPs, multiple linear regression was used to calculate odds ratios (ORs) with 95\% confidence intervals (95\% CIs). Two-tailed $P$-values $<0.05$ were considered statistically significant.

\section{Results}

\section{Characteristics of participants}

The maternal age in the study was from 21 to 42 years. A total of $321(70.4 \%)$ women were first-time mother and $20(4.4 \%)$ women delivered preterm birth $(<37$ weeks). In addition, 271 (59.4\%) women had vaginal delivery and others $(40.6 \%)$ delivered by cesarean section. Moreover, 102 (22.4\%) infants were breastfed exclusively, as shown in Table 1.

The maternal feeding anxiety was prevalent, and $61.4 \%$ (280/456) had anxiety symptom. Among those the prevalence of mild and severe FA symptom was $52.9 \%$ (241/456) and 8.6\% (39/456), respectively.

The feeding anxiety score (FAS) among 456 postpartum women was $41.02 \pm 8.02$ (mean $\pm \mathrm{SD}$ ). Differences in FAS and FA prevalence among groups categorized by sociodemographic characteristics were analyzed by oneway ANOVA, as shown in Table 2. In Table 2, the chi square test indicated the delivery mode was associated with postpartum feeding anxiety significantly $(P<0.05)$, but without significance if combining the mild and severe FA as one group (OR $=1.33,95 \% \mathrm{CI}$ : $0.90-1.96$ ). Furtherly comparing the mild and severe FA groups with negative group separately, the results showed the maternal mild FA prevalence with caesarean delivery was much higher than those with vaginal delivery $(60.0 \%$ vs $48.0 \%$, OR $=1.49,95 \%$ CI: $1.00-2.22, P<0.05$ ), but the prevalence of severe FA was not significantly different ( $5.4 \%$ vs $10.7 \%$, OR $=0.60,95 \%$ CI: $0.28-1.32, P>0.05$ ). So the study showed the CS delivery related with postpartum mild FA risk, but couldn't conclude the CS affect positively severe FA. A higher FAS was found in women with younger age, lower household income, first-time mother and preterm birth, but without statistical significance $(P>0.05)$. FA prevalence was associated with delivery mode $(P<0.05)$. 
Table 1 Characteristics of participants $(n=456)$

\begin{tabular}{|c|c|c|c|c|c|}
\hline Characteristics & $\mathbf{N}$ & Percentage (\%) & Characteristics & $\mathbf{N}$ & Percentage (\%) \\
\hline Maternal age (years) & & & \multicolumn{3}{|c|}{ Gestational age (weeks) } \\
\hline $21-27$ & 110 & 24.1 & $\geq 37$ & 436 & 95.6 \\
\hline $28-34$ & 277 & 60.7 & $<37$ & 20 & 4.4 \\
\hline$\geq 35$ & 69 & 15.1 & & & \\
\hline Educational degree & & & \multicolumn{3}{|l|}{ Delivery mode } \\
\hline$\leq$ High school & 170 & 37.3 & Vaginal & 271 & 59.4 \\
\hline Bachelor & 214 & 46.9 & Caesarean & 185 & 40.6 \\
\hline Postgraduate or above & 72 & 15.8 & & & \\
\hline Household income level & & & \multicolumn{3}{|c|}{ Exclusive breastfeeding } \\
\hline Low & 93 & 20.4 & Yes & 102 & 22.4 \\
\hline Middle & 202 & 44.3 & No & 354 & 77.6 \\
\hline High & 161 & 35.3 & \multicolumn{3}{|c|}{ Feeding anxiety symptom } \\
\hline Child-bearing number & & & Negative & 176 & 38.6 \\
\hline 1 & 321 & 70.4 & Mild & 241 & 52.9 \\
\hline$\geq 2$ & 135 & 29.6 & Severe & 39 & 8.6 \\
\hline
\end{tabular}

Table 2 Difference in FAS and FA prevalence in relation to different characteristics

\begin{tabular}{|c|c|c|c|c|c|c|c|c|}
\hline \multirow[t]{2}{*}{ Characteristics } & \multirow[t]{2}{*}{$\mathrm{N}$} & \multirow{2}{*}{$\begin{array}{l}\text { FAS } \\
(\text { mean } \pm \text { SD) }\end{array}$} & \multirow[t]{2}{*}{$P$ value } & \multicolumn{3}{|c|}{ Feeding anxiety $n(\%)$} & \multirow[t]{2}{*}{$P$ value } & \multirow[t]{2}{*}{$\mathrm{ORs}^{\mathrm{a}}(95 \% \mathrm{Cl})$} \\
\hline & & & & Negative( $\leq 38)$ & Mild(39 52) & Severe $(\geq 53)$ & & \\
\hline \multicolumn{9}{|l|}{ Maternal age (years) } \\
\hline $21-27$ & 110 & $41.71 \pm 8.27$ & 0.487 & $40(36.4 \%)$ & $60(54.5 \%)$ & $10(9.1 \%)$ & 0.830 & 1 \\
\hline $28-34$ & 277 & $40.94 \pm 8.12$ & & $105(37.9 \%)$ & $148(53.4 \%)$ & $24(8.7 \%)$ & & $0.94(0.59 \sim 1.48)$ \\
\hline$\geq 35$ & 69 & $40.23 \pm 8.45$ & & 31 (44.9\%) & $33(47.8 \%)$ & $5(7.2 \%)$ & & $0.70(0.38 \sim 1.29)$ \\
\hline \multicolumn{9}{|l|}{ Education degree } \\
\hline$\leq$ High school & 170 & $40.97 \pm 7.94$ & 0.951 & $63(37.1 \%)$ & $92(54.1 \%)$ & $15(8.8 \%)$ & 0.744 & 1 \\
\hline Bachelor & 214 & $41.13 \pm 8.31$ & & 81 (37.9\%) & $116(54.2 \%)$ & 17 (7.9\%) & & $0.97(0.64 \sim 1.47)$ \\
\hline Postgraduate or above & 72 & $40.79 \pm 8.59$ & & $32(44.4 \%)$ & $33(45.8 \%)$ & $7(9.7 \%)$ & & $0.74(0.42 \sim 1.29)$ \\
\hline \multicolumn{9}{|l|}{ Household income level } \\
\hline Low & 93 & $42.59 \pm 9.48$ & 0.111 & $33(35.5 \%)$ & $50(53.8 \%)$ & $10(10.8 \%)$ & 0.886 & 1 \\
\hline Middle & 202 & $40.73 \pm 8.12$ & & 78 (38.6\%) & $107(53.0 \%)$ & $17(8.4 \%)$ & & $0.87(0.52 \sim 1.46)$ \\
\hline High & 161 & $40.47 \pm 7.40$ & & 65 (40.4\%) & $84(52.2 \%)$ & $12(7.5 \%)$ & & $0.81(0.48 \sim 1.38)$ \\
\hline \multicolumn{9}{|l|}{ Parity } \\
\hline 1 & 321 & $41.13 \pm 8.40$ & 0.659 & $126(39.3 \%)$ & $163(50.8 \%)$ & $32(10.0 \%)$ & 0.170 & 1 \\
\hline$\geq 2$ & 135 & $40.76 \pm 7.74$ & & $50(37.0 \%)$ & $78(57.8 \%)$ & $7(5.2 \%)$ & & $1.10(0.73 \sim 1.63)$ \\
\hline \multicolumn{9}{|l|}{ Gestational age (weeks) } \\
\hline$\geq 38$ & 436 & $40.99 \pm 8.19$ & 0.767 & $168(38.5 \%)$ & $231(53.0 \%)$ & $37(8.5 \%)$ & 0.888 & 1 \\
\hline$<37$ & 20 & $41.55 \pm 8.59$ & & $8(40.0 \%)$ & $10(50.0 \%)$ & $2(10.0 \%)$ & & $0.94(0.38 \sim 2.35)$ \\
\hline \multicolumn{9}{|l|}{ Delivery mode } \\
\hline Vaginal & 271 & $41.06 \pm 8.42$ & 0.887 & $112(40.3 \%)$ & $130(48.0 \%)$ & $29(10.7 \%)$ & $0.019^{*}$ & 1 \\
\hline Caesarean & 185 & $40.95 \pm 7.88$ & & 64 (34.6\%) & 111 (60.0\%) & $10(5.4 \%)$ & & $1.33(0.90 \sim 1.96)$ \\
\hline
\end{tabular}

FAS Feeding anxiety score

${ }^{\text {a }}$ ORs value was calculated as combination of mild and severe anxiety (FAS $>38$ ) compared with negative (FA $\left.\leq 38\right)$ group

* $P<0.05$ 
Relations of maternal feeding anxiety with infant breastfeeding-related practices

The lower maternal FAS was significantly related with infant colostrum feeding $(40.86 \pm 8.02$ vs $44.74 \pm 11.33$, $P<0.05)$, but higher FAS was related with infant bottle feeding $(41.95 \pm 8.28$ vs $39.69 \pm 7.92, P<0.05)$ and outdoor sunshine exposure $(41.93 \pm 8.18$ vs. $40.27 \pm 8.16$, $P<0.05)$, as shown in Table 3 . The mothers with severe feeding anxiety (FAS $>53)$ were more likely to feed infants with bottle (ORs, 95\%CI: 2.41, $1.11 \sim 5.19$ ), compared with combination of mild and negative FA. There were not significant association between FA and exclusive breastfeeding $(P>0.05)$.

\section{Relations of maternal feeding anxiety with responsive feeding practices}

Differences in FAS between groups categorized by responsive feeding practices were analyzed by one-way
ANOVA and were shown in Table 4. However, the study found no significant relationship between responsive feeding practices and maternal FAS and FA prevalence $(P>0.05)$.

\section{Relations of maternal feeding anxiety with infant food refusal behaviors}

The higher FAS was associated with infant food refusal behaviors, the maternal scores whose infant "never", "rarely", "sometimes" and "often" spat out food when feeding were $39.86 \pm 8.02,41.47 \pm 8.18$, $41.36 \pm 7.44$ and $42.14 \pm 12.03$ increasingly $(P>0.05)$, and the FA prevalence was significantly different among groups $(P<0.05)$, as shown in Table 5 . The infants whose mother was identified as feeding anxiety were more likely to refuse opening the mouth when feeding $(P<0.05)$.

Table 3 Difference in maternal FAS and FA prevalence in relation to breastfeeding-related practices

\begin{tabular}{|c|c|c|c|c|c|c|c|c|}
\hline \multirow{2}{*}{$\begin{array}{l}\text { Breastfeeding- } \\
\text { related practices }\end{array}$} & \multirow[t]{2}{*}{$\mathbf{N}$} & \multirow{2}{*}{$\begin{array}{l}\text { FAS } \\
\text { (mean } \pm \text { SD) }\end{array}$} & \multirow[t]{2}{*}{$P$ value } & \multicolumn{3}{|c|}{ Feeding anxiety $n(\%)$} & \multirow[t]{2}{*}{$P$ value } & \multirow[t]{2}{*}{ ORs $(95 \% \mathrm{Cl})^{a}$} \\
\hline & & & & Negative( $\leq 38)$ & Mild(39 52) & Severe $(\geq 53)$ & & \\
\hline \multicolumn{9}{|c|}{$\begin{array}{l}\text { Touching breast in mother's arms within } \\
1 \mathrm{~h} \text { after delivery }\end{array}$} \\
\hline No & 197 & $41.00 \pm 8.22$ & 0.968 & $78(39.6 \%)$ & $102(51.8 \%)$ & $17(8.6 \%)$ & 0.920 & 1 \\
\hline Yes & 259 & $41.03 \pm 8.20$ & & 98 (37.8\%) & $161(62.2 \%)$ & $22(8.5 \%)$ & & $1.08(0.74 \sim 1.58)$ \\
\hline \multicolumn{9}{|l|}{ Colostrum feeding } \\
\hline No & 19 & $44.74 \pm 11.33$ & $0.043^{*}$ & $6(31.6 \%)$ & $10(52.6 \%)$ & $3(15.8 \%)$ & 0.376 & 1 \\
\hline Yes & 437 & $40.86 \pm 8.02$ & & 170 (38.9\%) & $231(52.9 \%)$ & $36(8.2 \%)$ & & $0.73(0.27 \sim 1.94)$ \\
\hline \multicolumn{9}{|c|}{$\begin{array}{l}\text { Feeding other non-milk food or liquid } \\
\text { before breastfeeding after delivery }\end{array}$} \\
\hline No & 272 & $41.19 \pm 7.89$ & 0.583 & $101(37.1 \%)$ & $148(54.4 \%)$ & $23(8.5 \%)$ & 0.707 & 1 \\
\hline Yes & 184 & $40.76 \pm 8.66$ & & $75(40.8 \%)$ & $93(50.5 \%)$ & $16(8.7 \%)$ & & $0.86(0.59 \sim 1.26)$ \\
\hline \multicolumn{9}{|c|}{ Exclusive breastfeeding } \\
\hline No & 354 & $40.99 \pm 8.14$ & 0.911 & $132(37.3 \%)$ & $194(54.8 \%)$ & $28(7.9 \%)$ & 0.272 & 1 \\
\hline Yes & 102 & $41.10 \pm 8.45$ & & $44(43.1 \%)$ & $47(53.9 \%)$ & $11(10.8 \%)$ & & $0.78(0.50 \sim 1.23)$ \\
\hline \multicolumn{9}{|c|}{ Feeding water, beverages or juice presently } \\
\hline No & 201 & $40.40 \pm 7.73$ & 0.156 & $78(38.8 \%)$ & $109(54.2 \%)$ & $14(7.0 \%)$ & 0.551 & 1 \\
\hline Yes & 255 & $41.50 \pm 8.54$ & & $98(38.4 \%)$ & $132(51.8 \%)$ & $25(9.8 \%)$ & & $1.02(0.70 \sim 1.49)$ \\
\hline \multicolumn{9}{|c|}{ Feeding infant formula } \\
\hline No & 261 & $40.74 \pm 8.11$ & 0.403 & $101(38.7 \%)$ & $142(54.4 \%)$ & $18(6.9 \%)$ & 0.327 & 1 \\
\hline Yes & 195 & $41.39 \pm 8.33$ & & 75 (38.5\%) & 99 (50.8\%) & $21(10.8 \%)$ & & $1.01(0.69 \sim 1.48)$ \\
\hline \multicolumn{9}{|l|}{ Bottle feeding } \\
\hline No & 188 & $39.69 \pm 7.92$ & $0.004^{*}$ & 79 (42.0\%) & $100(53.2 \%)$ & $9(4.8 \%)$ & $0.043^{*}$ & 1 \\
\hline Yes & 268 & $41.95 \pm 8.28$ & & 97 (36.2\%) & $141(52.6 \%)$ & $30(11.2 \%)$ & & $1.28(0.87 \sim 1.87)$ \\
\hline \multicolumn{9}{|c|}{ Outdoor sunshine exposure } \\
\hline No & 251 & $40.27 \pm 8.16$ & $0.032^{*}$ & $106(42.2 \%)$ & $125(49.8 \%)$ & $20(8.0 \%)$ & 0.210 & 1 \\
\hline Yes & 205 & $41.93 \pm 8.18$ & & $70(34.1 \%)$ & $116(56.6 \%)$ & 19 (9.3\%) & & $1.41(0.96 \sim 2.07)$ \\
\hline
\end{tabular}

FAS Feeding anxiety score

${ }^{a}$ ORs value was calculated as combination of mild and severe anxiety (FAS $>38$ ) compared with negative (FA $\left.\leq 38\right)$ group * $P<0.05$ 
Table 4 Difference in maternal FAS and FA prevalence in relation to responsive feeding practices

\begin{tabular}{|c|c|c|c|c|c|c|c|c|}
\hline \multirow{2}{*}{$\begin{array}{l}\text { Responsive } \\
\text { feeding }\end{array}$} & \multirow[t]{2}{*}{$\mathbf{N}$} & \multirow{2}{*}{$\begin{array}{l}\text { FAS } \\
(\text { mean } \pm S D)\end{array}$} & \multirow[t]{2}{*}{$P$ value } & \multicolumn{3}{|c|}{ Feeding anxiety $n(\%)$} & \multirow[t]{2}{*}{$P$ value } & \multirow[t]{2}{*}{$\operatorname{ORs}(95 \% \mathrm{Cl})^{\mathrm{a}}$} \\
\hline & & & & Negative $(\leq 38)$ & Mild(39 52) & Severe $(\geq 53)$ & & \\
\hline \multicolumn{9}{|c|}{ Feeding as more as possible } \\
\hline Never & 85 & $40.58 \pm 8.02$ & 0.868 & $33(38.8 \%)$ & $45(52.9 \%)$ & $7(8.2 \%)$ & 0.996 & 1 \\
\hline Rarely & 74 & $40.80 \pm 8.83$ & & $28(37.8 \%)$ & $40(54.1 \%)$ & $6(8.1 \%)$ & & $1.04(0.55 \sim 1.98)$ \\
\hline Sometimes & 111 & $40.82 \pm 8.10$ & & $44(39.6 \%)$ & $59(53.2 \%)$ & $67(60.4 \%)$ & & $0.97(0.54 \sim 1.72)$ \\
\hline Often & 132 & $41.68 \pm 8.03$ & & $71(38.2 \%)$ & $97(52.2 \%)$ & $82(62.1 \%)$ & & $1.03(0.61 \sim 1.74)$ \\
\hline \multicolumn{9}{|c|}{ Perception of infant hungry and satiety signs } \\
\hline Never & 198 & $41.13 \pm 8.41$ & 0.171 & $74(37.4 \%)$ & $107(54.0 \%)$ & $17(8.6 \%)$ & 0.934 & 1 \\
\hline Rarely & 224 & $41.19 \pm 8.20$ & & 87 (38.8\%) & $116(51.8 \%)$ & $21(9.4 \%)$ & & $0.94(0.63-1.39)$ \\
\hline Sometimes & 21 & $40.62 \pm 6.04$ & & $8(38.1 \%)$ & $12(57.1 \%)$ & $1(4.8 \%)$ & & $0.97(0.38-2.45)$ \\
\hline Often & 4 & $31.00 \pm 8.83$ & & 7 (53.8\%) & $6(46.2 \%)$ & $0(0.0 \%)$ & & $0.51(0.17-1.58)$ \\
\hline \multicolumn{9}{|c|}{ Fixed-time feeding } \\
\hline Never & 78 & $40.21 \pm 7.83$ & 0.573 & 33 (42.3\%) & $42(53.8 \%)$ & $3(3.8 \%)$ & 0.649 & 1 \\
\hline Rarely & 86 & $41.87 \pm 8.03$ & & $30(34.9 \%)$ & $48(55.8 \%)$ & $8(9.3 \%)$ & & $1.37(0.73 \sim 2.57)$ \\
\hline Sometimes & 97 & $41.62 \pm 8.72$ & & 34 (35.1\%) & $54(55.7 \%)$ & $9(9.3 \%)$ & & $1.36(0.74 \sim 2.51)$ \\
\hline Often & 130 & $40.42 \pm 7.90$ & & 79 (40.5\%) & 97 (49.7\%) & 19 (9.7\%) & & $1.08(0.63 \sim 1.83)$ \\
\hline
\end{tabular}

FAS Feeding anxiety score

${ }^{a}$ ORs value was calculated as combination of mild and severe anxiety (FAS $>38$ ) compared with negative (FA $\left.\leq 38\right)$ group * $P<0.05$

\section{Multivariate analysis \}

Multivariate linear regression was conducted to analyze the relations of maternal feeding anxiety with infant feeding behaviors. Variables included in the final model were determined by backward deletion method step by step with $P<0.05$. The likelihood ratio $F$ test was used for overall test of the model with result of $F=5.011, P<$ 0.001. After adjusting for maternal age, education level, household income, child-bearing number, gestational age and delivery mode, maternal FAS was positively related to infant bottle feeding and outdoor sunshine exposure practice $(P<0.05)$, and negatively related to household income level, as shown in Table 6.

\section{Discussion}

Our study indicated the maternal feeding anxiety was prevalent in China and $61.4 \%$ of women at $0-3$ months postpartum had feeding anxiety symptoms, with $8.6 \%$ being severe anxiety. Maternal postpartum feeding anxiety was associated with negative infant feeding practices, like bottle feeding and infant food refusal behaviors. The results showed maternal mental health may be an important factor affecting child nutrition and health. Negative emotions during feeding may affect the maternal cerebral cortex, hypothalamus, and pituitary function, decrease secretion of prolactin and oxytocin, eventually reduce the breast milk secretion $[6,9]$.

Pregnancy and therefore lactation are important life events for women, during which their physiological and psychological state, as well as social status undergo considerable changes. The immediate change in life roles and new responsibilities after childbirth may be pressure provoking. Therefore, postpartum women are susceptible to anxiety, fear, and even depression [10]. Perinatal anxiety and depression are most common mental health problems in women. A systematic review summarized the prevalence and determinants of non-psychotic common perinatal mental disorders (CPMDs) in World Bank categorized low- and lower-middle-income countries, showed weighted mean prevalence was $15.6 \%$ (95\% CI: $15.4-15.9)$ antenatally and $19.8 \%(19.5-20.0)$ postnatally, and the pooled prevalence of perinatal depression ranged from 5.2 to $32.9 \%$ during pregnancy and $4.9-$ $59.4 \%$ after child birth [1]. In China the prevalence of postnatal anxiety or depression varies from 2.2 to $43.6 \%$ [11-14]. A systematic review of postpartum depression prevalence published during 1996-2012 showed the pooled prevalence was $14.7 \%$ (13.1-16.3\%), increasing from the east of China to the west [15].

Given postpartum anxiety has been noted as having independent effects just as postpartum depression, and significant comorbidity has also been noted between postpartum anxiety and depression, it is surprising that so little research has been conducted on postpartum anxiety, compared with postpartum depression. A narrative review showed [5] the prevalence of postpartum anxiety (ranged from 13 to 40\%) has varied according to the definition, the anxiety scale used, the cut-off scores on the scales, the severity of anxiety, the timing of the assessment, the recruitment sample and the origin (country) 
Table 5 Difference in maternal FAS and FA prevalence in relation to infant food refusal behaviors

\begin{tabular}{|c|c|c|c|c|c|c|c|c|}
\hline \multirow{2}{*}{$\begin{array}{l}\text { Infant food } \\
\text { refusal behaviors } \\
\text { when feeding }\end{array}$} & \multirow[t]{2}{*}{$\mathbf{N}$} & \multirow{2}{*}{$\begin{array}{l}\text { FAS } \\
(\text { mean } \pm S D)\end{array}$} & \multirow[t]{2}{*}{$P$ value } & \multicolumn{3}{|c|}{ Feeding anxiety $n(\%)$} & \multirow[t]{2}{*}{$P$ value } & \multirow[t]{2}{*}{$\operatorname{ORs}(95 \% \mathrm{Cl})^{\mathrm{a}}$} \\
\hline & & & & Negative( $\leq 38)$ & Mild(39 52) & Severe $(\geq 53)$ & & \\
\hline \multicolumn{9}{|l|}{ Spitting out food } \\
\hline Never & 126 & $39.86 \pm 8.02$ & 0.436 & $54(42.9 \%)$ & $66(52.4 \%)$ & $6(4.8 \%)$ & $0.001^{*}$ & 1 \\
\hline Rarely & 150 & $41.47 \pm 8.18$ & & $58(38.7 \%)$ & $73(48.7 \%)$ & 19 (12.7\%) & & $1.19(0.73 \sim 1.93)$ \\
\hline Sometimes & 148 & $41.36 \pm 7.44$ & & 47 (31.8\%) & $93(78.2 \%)$ & $8(5.4 \%)$ & & $1.61(0.98 \sim 2.64)$ \\
\hline Often & 28 & $42.14 \pm 12.03$ & & $17(53.1 \%)$ & $9(28.1 \%)$ & $6(18.8 \%)$ & & $0.66(0.30 \sim 1.44)$ \\
\hline \multicolumn{9}{|c|}{ Refusing to open mouth } \\
\hline Never & 250 & $40.51 \pm 7.77$ & 0.244 & $93(37.2 \%)$ & $144(57.6 \%)$ & $13(5.2 \%)$ & $0.026^{*}$ & 1 \\
\hline Rarely & 147 & $41.23 \pm 8.81$ & & $64(43.5 \%)$ & $63(42.9 \%)$ & $20(13.6 \%)$ & & $0.77(0.51 \sim 1.61)$ \\
\hline Sometimes & 54 & $42.93 \pm 8.34$ & & $17(31.5 \%)$ & $31(28.5 \%)$ & $6(11.1 \%)$ & & $1.29(0.69 \sim 2.42)$ \\
\hline Often & 5 & $39.40 \pm 8.08$ & & $2(40.0 \%)$ & $3(60.0 \%)$ & $0(0.0 \%)$ & & $0.89(0.15 \sim 5.42)$ \\
\hline \multicolumn{9}{|l|}{ Turning heads aside } \\
\hline Never & 205 & $41.17 \pm 7.87$ & 0.809 & $70(34.1 \%)$ & $120(58.5 \%)$ & $15(7.3 \%)$ & 0.354 & 1 \\
\hline Rarely & 155 & $40.63 \pm 8.52$ & & 67 (43.2\%) & $71(45.8 \%)$ & $17(11.0 \%)$ & & $0.68(0.44 \sim 1.05)$ \\
\hline Sometimes & 89 & $41.48 \pm 8.50$ & & $36(40.4 \%)$ & 46 (51.7\%) & 7 (7.9\%) & & $0.76(0.46 \sim 1.27)$ \\
\hline Often & 7 & $39.43 \pm 7.70$ & & $3(42.9 \%)$ & $4(51.7 \%)$ & $0(0.0 \%)$ & & $0.69(0.15 \sim 3.18)$ \\
\hline \multicolumn{9}{|l|}{ Crying } \\
\hline Never & 201 & $40.94 \pm 7.90$ & 0.955 & 75 (37.3\%) & 111 (55.2\%) & $15(7.5 \%)$ & 0.746 & 1 \\
\hline Rarely & 168 & $40.90 \pm 8.59$ & & 68 (40.5\%) & 83 (49.4\%) & 17 (10.1\%) & & $0.88(0.57 \sim 1.33)$ \\
\hline Sometimes & 82 & $41.39 \pm 7.90$ & & 31 (37.8\%) & 45 (54.9\%) & $6(7.3 \%)$ & & $0.98(0.58 \sim 1.66)$ \\
\hline Often & 5 & $42.20 \pm 13.41$ & & $2(40.0 \%)$ & $2(40.0 \%)$ & $1(20.0 \%)$ & & $0.89(0.15 \sim 5.47)$ \\
\hline
\end{tabular}

FAS Feeding anxiety score

${ }^{a}$ ORs value was calculated as combination of mild and severe anxiety (FAS $>38$ ) compared with negative (FA $\leq 38$ ) group

* $P<0.05$

of research. In this study, the Self-rating Feeding Anxiety Scale (SFAS) was used to assess the maternal postpartum anxiety, which was developed specifically to tap postnatalspecific anxiety-feeding anxiety, and reliability and validity has been tested [13]. Besides assessment tools, the cultural and socio-economic settings, sample sizes, cutoff point used, and timing of assessment affect prevalence. In this study the maternal feeding anxiety was assessed at $0 \sim 3$ months postpartum, which was during the Chinese traditional "doing the month" period, both limited socialization and family support could worsen maternal mental problems and lead to higher anxiety symptom prevalence. In fact, the severe
FA prevalence was only $8.6 \%$ (FAS $>52$ ), that was similar to other studies. In order to achieve reliable results globally, there is a need to establish widely accepted assessment tools, cutoff scores, and timing of assessment.

Systematic review showed the correlates factors for postpartum anxiety included demographic factors, childbirth experiences, social support and history of psychiatric and psychological problems [1]. The demographic risk factors for postnatal anxiety include being a young mother, having more education and being employed. Childbirth risk factors include being primiparous in one sample and multiparous in another, caesarean delivery,

Table 6 Multivariate analysis of maternal feeding anxiety as dependent variable

\begin{tabular}{|c|c|c|c|c|c|}
\hline \multirow[t]{2}{*}{ Independent variable } & \multirow[t]{2}{*}{$\beta \mathbf{i}$} & \multirow[t]{2}{*}{$T$} & \multirow[t]{2}{*}{$P$ value } & \multicolumn{2}{|l|}{$95 \% \mathrm{Cl}$} \\
\hline & & & & Lower limit & Upper limit \\
\hline Constant & 42.820 & 10.139 & $<0.001$ & 34.520 & 51.119 \\
\hline Household income level & -1.083 & -2.097 & 0.037 & -2.098 & -0.068 \\
\hline Colostrum feeding & -3.656 & -1.934 & 0.054 & -7.370 & 0.058 \\
\hline Bottle feeding & 2.327 & 3.022 & 0.003 & 0.814 & 3.840 \\
\hline Outdoor sunshine exposure & 1.824 & 2.410 & 0.016 & 0.337 & 3.311 \\
\hline Refusing to open mouth & 0.846 & 1.655 & 0.099 & -0.158 & 1.849 \\
\hline
\end{tabular}

$\mathrm{F}=5.011, P<0.001$ 
fear of the birth and of death during delivery, lack of control during labor, low self-confidence for the delivery and the delivery staff, and premature delivery. Social support problems include the lack of family support, marital/family conflict, and social health issues. Psychiatric history risk factors include prenatal depression and anxiety. Several studies have concluded that preterm birth and cesarean section are independent risk factors of postpartum anxiety [16-18] We found a higher FAS in women with younger age, lower household income, first-time mother and preterm birth, but without statistical significance $(P>0.05)$, and FA prevalence was associated with delivery mode $(P<0.05)$. Trumello et al. [19] found that mother-child separation caused by the need for specialized care of premature infants may exert a negative effect on the mothers' emotional state; thus, women who give birth to premature infants are more likely to develop postpartum anxiety. A Chinese study [20] found that postpartum women aged $\geq 35$ were more prone to anxiety or depression. Ye et al. (2014) [21] reported a higher prevalence of postpartum anxiety among mothers with higher education levels. Zhang et al. [11] found that women were more vulnerable to feeding anxiety with lower household income. Bina R et al. (2017) [22] found that postpartum women with lower incomes reported more symptoms of anxiety. And another study showed that postpartum women's perceived difficulty managing on household income was associated with anxiety symptomatology [23]. Other studies have suggested that social psychological factors, such as emotional support from family and society, and various expectations of the sex of the newborn also considerably affected the mother's feelings during feeding [11, 24]. Because of the limited data we haven't analyzed the relations of FA with family support and history of psychiatric and psychological problems, that is the study limitation.

Postpartum anxiety are always associated with unpleasant emotional experiences, such as worry, fear, irritability, and frustration, that can last for a few weeks or even longer [25]. Maternal anxiety during feeding may eradicate any willingness to breastfed and even affect the composition of the breast milk, ultimately affecting the growth and development of infant. The systematic literature review included negative effects on breast-feeding, bonding, mother-infant interactions, infant temperament, sleep, mental development, health and internalizing in infants and on conduct disorder in adolescents, based on structured clinical interviews and behavior observations [1].

Our study indicated higher FA risk seemed to be found in women who did not feed infant colostrum or fed infant with bottle. This may be explained by that mothers who fed infant colostrum could generally let baby touch breast in mother's arms within $1 \mathrm{~h}$ after delivery, which promoted emotional communication between mother and infant and helped breastfeeding. However bottle feeding might reduce mother-child contact, and hinder emotional communication during feeding. Furthermore, bottle feeding led to insufficient breast milk secretion and subsequent interruption of breastfeeding, which diminished maternal confidence in infant feeding. On the other hand, bottle feeding might help mothers control the milk intake of infants, and mothers with FA may prefer bottle feeding to breastfeeding. Lower FA prevalence was found in women who breastfed exclusively but without statistical significance. These findings are supported by two studies, in which mothers who breastfed are less likely to develop FA, as compared to mothers who chose formula feeding, and mothers suffered from FA does not emerge favorable persistence signs associated with breastfeeding [26, 27].

Moreover, mothers also had a significantly higher FAS if their babies had been exposed to sunshine outdoors. A possible explanation may be that mothers with FA might be more cautious about the infant's health so they spent more time with their infants outdoors to synthesize vitamin D. Infant food refusal behaviors may reduce maternal confidence, and induce mother's anxiety about infants' nutrient intake and development. But above results needed to be confirmed by more research in the future, because the limitations of sample size and sampling method decreased the power of test.

Despite our results and the mounting evidence indicating high prevalence of maternal mental health and its adverse impact on infant feeding practices, the maternal mental health problems has not been focused. Consequently the treatment gap for mental illness is large, accounting for $76-85 \%$ patients with mental health not receiving intervention. Studies show that psychosocial, educational, and supportive interventions are effective in improving maternal mental health. That will be our next research direction, to explore the effective intervention strategy to improve maternal mental health and early child development. And theoretically the anesthesia type during delivery would have an influence on breastfeeding, but regrettably the related information has not been collected in this study. In the future, if possible we will investigate the data retrospectively and compensatively in the other relevant investigation.

Several limitations in our study deserved acknowledgements, like convenient sampling, small sample size, unpopular assessment tool, related factors (family support) data deficiency, etc.

\section{Conclusion}

Maternal FA prevalence were $61.4 \%$ (FAS $>38$ ) with severe FA being $8.6 \%($ FAS $>52)$ at $0-3$ months postpartum. Maternal postpartum feeding anxiety was associated with some infant feeding practices, including bottle feeding and infant food refusal behaviors. 


\section{Supplementary Information}

The online version contains supplementary material available at https:/doi. org/10.1186/s12884-020-03483-w.

Additional file 1. Questionnaire of assessment of feeding anxiety.

Additional file 2. Questionnaire of infant feeding practices (FPS) investigation.

Additional file 3. Questionnaire of demographic characteristics.

\section{Abbreviations}

FA: Feeding Anxiety; FPs: Feeding Practices; FAS: Feeding Anxiety Scores; SFAS: Self-rating Feeding Anxiety Scale; ORs: Odds Ratios; Cl: Confidence Intervals

\section{Acknowledgements}

We greatly acknowledge the support of local maternal and child health care institutions in 7 cities. We thank all mothers for their participation and support. We thank all teammates for their assistance in the various stages of data collection, data input and supervision of every procedure.

\section{Authors' contributions}

SJ, ZYM and ZWl analyzed the data and wrote the manuscript. And LYJ, LNN, LT, SX, DZY, ZYC, PLN and JW contributed to collect data and help finish the study. All authors reviewed and approved the final manuscript.

\section{Funding}

This research received no external funding.

\section{Availability of data and materials}

The datasets used and/or analyzed during the current study are available from the corresponding author on reasonable request.

\section{Ethics approval and consent to participate}

This study was reviewed and approved by the Peking University Institutional Review Board. Informed written consent was obtained from all mother participants.

\section{Consent for publication}

Not applicable.

\section{Competing interests}

The authors declare that they have no competing interests.

\section{Author details}

${ }^{1}$ Department of Nutrition and Food Hygiene, Peking University School of Public Health; National Health Commission Key Laboratory of Reproductive Health, Beijing 100191, China. ${ }^{2}$ Shijingshan District Center for Diseases Prevention and Control, Beijing 100043, China. ${ }^{3}$ Shunyi District Center for Diseases Prevention and Control, Beijing 101300, China. ${ }^{4}$ Peking-Ausnutria Maternal and Infant Nutrition Research Center, Beijing 100191, China.

\section{Received: 2 February 2020 Accepted: 8 December 2020}

Published online: 14 December 2020

\section{References}

1. Fisher J, Cabral de Mello M, Patel V, Rahman A, Tran T, Holton S, et al. Prevalence and determinants of common perinatal mental disorders in women in low- and lower-middle-income countries: a systematic review. Bull World Health Organ. 2012;90(2):139G-49G.

2. Dennis C-L, Brown HK, Wanigaratne S, Fung K, Vigod SN, Grigoriadis S, et al. Prevalence, incidence, and persistence of postpartum depression, anxiety, and comorbidity among Chinese immigrant and nonimmigrant women: a longitudinal cohort study. Can J Psychiatr. 2018;63(1):44-53.

3. Reck C, Noe D, Gerstenlauer J, Stehle E. Effects of postpartum anxiety disorders and depression on maternal self-confidence. Infant Behav Dev. 2012;35(2):264-72.

4. Sharifi F, Nouraei S, Shahverdi E. The relation of pre and postnatal depression and anxiety with exclusive breastfeeding. Electron Physician. 2016:8(11):3234-9.
5. Field T. Postnatal anxiety prevalence, predictors and effects on development: a narrative review. Infant Behav Dev. 2018;51:24-32.

6. Lara-Cinisomo S, McKenney K, Di Florio A, Meltzer-Brody S. Associations between postpartum depression, breastfeeding, and oxytocin levels in Latina mothers. Breastfeed Med. 2017;12(7):436-42.

7. Yongjin L. Design of of maternal feeding anxiety evaluation scale. Nutrition and diabetes complications- - the 16th academic conference of Danone nutrition center. Wuhan: Danone Institute; 2013.

8. Yongjin $L$, Junbo W, Wenli Z, Jinshuan $X$, Lingyan $Z$, Yuan $Z$, et al. A Preliminary Study of the Evaluation of Feeding Anxiety and Its Influence on Infant Eating Sentiment and Emotion, The 13th National Nutrition Science Conference of Chinese Nutrition Society and Global Chinese Nutrition Scientists Conference. Beijing: Chinese Nutrition Society; 2017.

9. Pawluski JL, Lonstein JS, Fleming AS. The neurobiology of postpartum anxiety and depression. Trends Neurosci. 2017:40(2):106-20.

10. Ling $Z$, Ning $L$, Juncai $H$. Comparison of correlative influencing factors of Mothers' anxiety and depression during feeding period. Jiangsu Health Care. 2014;16(05):49-51.

11. Chunxiang Z, Yuxia G, Lin L. Study of the correlation between postpartum anxiety and socioeconomic status among caesarean section women. Chinese Matern Child Health. 2017;32(16):3905-7.

12. Hui W, Yu D, Yijin Z, Weidong W. Investigation of Postpartum Anxiety of Women of Childbearing Age in Henan Province. Modern Prev Med. 2016; 43(01):57-9+98.

13. Ruixia A. Investigation and analysis of postpartum anxiety and depression of delivery women with idiopathic diseases. Nurs Pract Res. 2018;15(06):71-2.

14. Xu D, Yonglin L, Haoye X, Liang W, Wenli Z. Study on the distribution of feeding anxiety among 522 delivery women. Matern Child Health Care China. 2016;31(19):4033-6.

15. Yaorong Q, Xiaoying Y. Prevalence of postpartum depression in China: a systematic analysis. Chinese J Pract Nurs. 2013;29(12):1-3.

16. Clout D, Brown R. Sociodemographic, pregnancy, obstetric, and postnatal predictors of postpartum stress, anxiety and depression in new mothers. J Affect Disord. 2015;188:60-7.

17. Farr SL, Dietz PM, O'Hara MW, Burley K, Ko JY. Postpartum anxiety and comorbid depression in a population-based sample of women. J Women's Health (Larchmt). 2014:23(2):120-8.

18. Li W, Yan Z, Hui X, Qiping L. Investigation and analysis of postpartum anxiety of mothers of premature infants. Shanghai Nurs. 2017;17(01):49-51.

19. Trumello C, Candelori C, Cofini M, Cimino S, Cerniglia L, Paciello M, et al. Mothers' depression, anxiety, and mental representations after preterm birth: a study during the Infant's hospitalization in a neonatal intensive care unit. Front Public Health. 2018;6:359.

20. Yan C. Correlative influencing factors and preventive countermeasures for causing postpartum anxiety and depression. Med Theory Pract. 2015:28(22):3158-9.

21. Biqing $Y$. Analysis of the reasons and nursing countermeasures of postpartum anxiety and depression. Qilu Nurs J. 2014;20(02):63-6.

22. Bina R, Harrington D. Differential predictors of postpartum depression and anxiety: the Edinburgh postnatal depression scale Hebrew version two factor structure construct validity. Matern Child Health J. 2017; 21(12):2237-44

23. Dennis $\mathrm{CL}$, Falah-Hassani K, Brown HK, Vigod SN. Identifying women at risk for postpartum anxiety: a prospective population-based study. Acta Psychiatr Scand. 2016;134(6):485-93.

24. Navarrete LE, Lara-Cantú MA, Navarro C, Gómez ME, Morales F. Psychosocial factors predicting postnatal anxiety symptoms and their relation to symptoms of postpartum depression. Rev Investig Clin. 2012; 64(6 Pt 2):625-33.

25. Sriraman NK, Pham D-Q, Kumar R. Postpartum depression: what do pediatricians need to know? Pediatr Rev. 2017:38(12):541-51.

26. Dias CC, Figueiredo B. Breastfeeding and depression: a systematic review of the literature. J Affect Disord. 2015;171:142-54.

27. Fallon V, Groves R, Halford JCG, Bennett KM, Harrold JA. Postpartum anxiety and infant-feeding outcomes. J Hum Lact. 2016:32(4):740-58.

\section{Publisher's Note}

Springer Nature remains neutral with regard to jurisdictional claims in published maps and institutional affiliations. 